\title{
Ankylosing spondylitis and the risk of cancer
}

\author{
CHIH-CHENG CHANG ${ }^{1,2}$, CHENG-WEI CHANG $^{3}$, PHUNG-ANH ALEX NGUYEN ${ }^{4}$, TZU-HAO CHANG ${ }^{5}$, \\ YA-LING SHIH ${ }^{6}$, WEN-YING CHANG ${ }^{7}$, JORNG-TZONG HORNG $^{8}$, OSCAR KUANG-SHENG LEE ${ }^{9,10}$ \\ and JENNIFER HUI-CHUN HO${ }^{11}$
}

\begin{abstract}
${ }^{1}$ Department of Internal Medicine, College of Medicine, Taipei Medical University, Taipei 110; ${ }^{2}$ Division of Pulmonary Medicine, Department of Internal Medicine, Shuang Ho Hospital, Taipei Medical University, Taipei 235 ; ${ }^{3}$ Department of Information Management, Hsing Wu University, New Taipei $244 ;{ }^{4}$ College of Medical Science \& Technology; ${ }^{5}$ Graduate Institute of Biomedical Informatics, Taipei Medical University, Taipei 110; ${ }^{6}$ Department of Nursing, En Chu Kong Hospital, New Taipei 237; ${ }^{7}$ Department of Electrical Engineering, National Cheng-Kung University, Tainan 701; ${ }^{8}$ Department of Biomedical Informatics, Asia University, Taichung 413; ${ }^{9}$ Institute of Clinical Medicine, National Yang-Ming University, Taipei 112; ${ }^{10}$ Department of Orthopaedic Surgery, Taipei City Hospital, Taipei $103 ;{ }^{11}$ Center for Stem Cell Research and Clinical Trial, Wan Fang Hospital, Taipei Medical University, Taipei 116, Taiwan, R.O.C.
\end{abstract}

Received August 23, 2015; Accepted February 13, 2017

DOI: $10.3892 / \mathrm{ol} .2017 .6368$

\begin{abstract}
Cancer is a multifactorial disease, and imbalances of the immune response and sex-associated features are considered risk factors for certain types of cancer. The present study aimed to assess whether ankylosing spondylitis (AS), an immune disorder that predominantly affects young adult men, is associated with an increased risk of cancer. Using the Taiwan National Health Insurance Research Database, a cohort of patients diagnosed with AS between 2000 and 2008 who had no history of cancer prior to enrollment was established $(n=5,452)$. Age- and sex-matched patients without AS served as controls $(n=21,808)$. The results revealed that the overall incidence of cancer was elevated in patients with AS [standardized incidence ratio (SIR), 1.15; 95\% confidence interval (CI), 1.03-1.27]. AS carried an increased risk of hematological malignancy in both sexes, colon cancer in females and bone and prostate cancer in males. Young patients with AS ( $\leq 35$ years) and patients with a Charlson comorbidity index $(\mathrm{CCI}) \geq 2$ experienced a higher incidence of cancer (males, SIR 1.92, and 95\% CI 1.04-3.26; females, SIR 2.00 and $95 \%$ CI 1.46-5.50). The cancer risk was increased during the first 3 years following the diagnosis of AS (SIR 1.49, 95\%
\end{abstract}

Correspondence to: Professor Jennifer Hui-Chun Ho, Center for Stem Cell Research and Clinical Trial, Wan Fang Hospital, Taipei Medical University, $250 \mathrm{Wu}-\mathrm{Hsing}$ Street, Taipei 116, Taiwan, R.O.C.

E-mail:wh9801@yahoo.com.tw

Professor Oscar Kuang-Sheng Lee, Department of Orthopedic Surgery, Taipei City Hospital, 145 Zhengzhou Road, Datong, Taipei City 103, Taiwan, R.O.C.

E-mail: oscarlee9203@gmail.com

Key words: age groups, ankylosing spondylitis, cancer, cohort, comorbidity, sex
CI 1.29-1.71), and overall cancer-free survival was significantly decreased in patients with AS patients of both sexes $(\mathrm{P}<0.0001)$. Therefore, AS was found to be associated with an increased risk of cancer. All AS patients must be screened for hematological malignancies, for prostate and bone cancer in males, and for colon cancer in females, particularly younger patients with a $\mathrm{CCI} \geq 2$.

\section{Introduction}

Ankylosing spondylitis (AS) is a disorder in which an abnormal immune response triggers chronic inflammation in large joints, particularly at the sacroiliac junction (1). AS is a human leucocyte antigen (HLA)-B27-related joint disorder that predominantly affects adolescent men and initially presents as lower back pain $(1,2)$. More than $90 \%$ of Chinese AS patients are HLA-B27 positive, with the predominant subtype being HLA-B*2704 (3). The axial spine and joints are most commonly affected in patients with AS; however, the disease also affects the heart, lungs, eyes, and colon, leading to complications such as uveitis (4), iridocyclitis (5), osteoporosis (6) and inflammatory bowel disease (7). Treatments for AS include non-steroidal anti-inflammatory drugs, tumor necrosis factor-alpha (TNF- $\alpha$ ) inhibitors and physiotherapy to alleviate joint pain and stiffness. Surgical intervention is an option in patients with advanced AS, and the prognosis is associated with the disease severity (1).

In addition, cancer is a leading cause of mortality and a major public health concern in Taiwan (8). Tumorigenesis is a multifactorial process, and chronic inflammation promotes the development of cancer. Epidemiological studies have revealed that patients with chronic inflammation are at increased risk of developing cancer $(9,10)$. Pathologically, chemokines, cytokines and prostaglandins are able to shift the microenvironment of an organ from a healthy to a dysplastic state, which favors malignant changes in affected cells (10). Elevated serum interleukin (IL)-1, IL-6, and TNF- $\alpha$ levels 
trigger inflammatory reactions in patients with spondyloarthritis (including AS) (11).

Thus, the potential association between AS and cancer is controversial. Furthermore, little is known about whether $\mathrm{AS}$ in men and women differentially affects the incidence of specific cancer types or cancer survival. In this retrospective cohort study, data from patients with AS and patients without AS collected between 2000 and 2008 from the Taiwanese National Health Insurance Research Database (NHIRD), a national population-based medical database, was analyzed. The characteristics, comorbidities, cancer risk and cancer-free survival probability of patients with AS were compared with those of sex- and age-matched patients without AS.

\section{Patients and methods}

Data Sources. The National Health Insurance is a single-payer system that was created by integrating 13 public insurance systems in 1995 to serve all residents of Taiwan. Approximately $99 \%$ of the estimated population of 23.7 million residents of Taiwan has been covered by this health-care insurance program since 1999 (12). The Taiwan Department of Health has authorized the National Health Research Institute (NHRI) to manage the claims data of the National Health Insurance Research Database (NHIRD). Data in this study were generated from records of 1 million insured people that were released by the NHRI. Medical records of outpatients and inpatients were collected between January 1998 and December 2008, and data from each patient was able to be linked to their longitudinal medical history using an encrypted unique personal identifier. The personal identifications were scrambled to ensure patient confidentiality and to prevent unethical use of the claims data. The present study confirmed that all the data were de-identified and analyzed anonymously. The study was approved by the Taipei Medical University Joint Institutional Review Board (TMU-JIRB-201404109).

Study population. In this retrospective cohort study, the patients were classified either as having AS or as non-AS (Fig. 1). All individuals newly diagnosed with AS [International Classification of Disease, Clinical Modification, Ninth Revision (ICD-9-CM) codes 720.0 (13)] with at least three outpatient visits between 2000 and 2008 recorded in their medical records were included. Patients who were diagnosed with AS before 2000, were of unknown sex, were aged younger than 16 years and were diagnosed with any cancer prior to AS were excluded. The individuals without an AS diagnosis during the 10 years assessed in the study served as controls. For each case, four controls were randomly selected among all of the individuals in the sample population who were matched by age and sex at the time of AS diagnosis. All of the subjects in this study were followed up until a cancer diagnosis was made, until the subject was censored due to loss to follow-up, insurance termination, mortality or until the end of 2008 .

As the likelihood for developing cancer may be confounded by competing risks, comorbidities that may be associated with mortality based on diagnostic codes from outpatient datasets identified prior to the outcome of interest were evaluated.

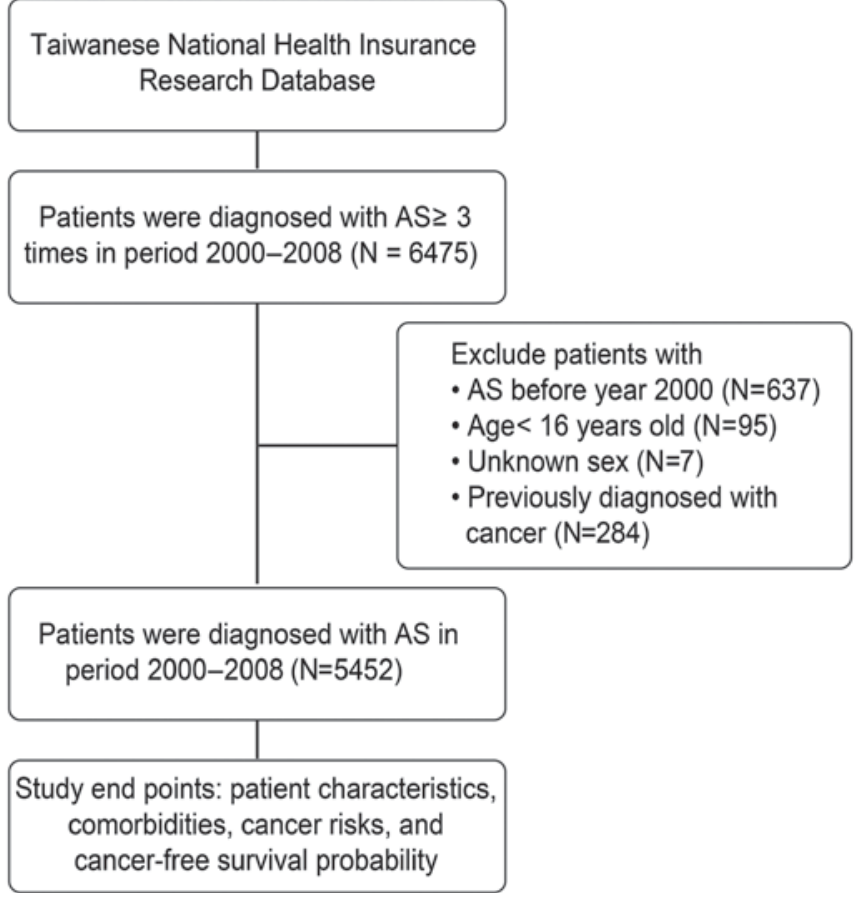

Figure 1. A flowchart of the study scheme. AS, ankylosing spondylitis.

The other diseases comprised iridocyclitis (ICD9-CM codes 364.0, 364.01, 364.03, 364.10 and 364.11), inflammatory bowel disease (ICD9-CM codes 555 without 560.9, and 556 without 560.9), osteoporosis (ICD9-CM codes 733.0 to 733.01, 733.02, 733.03 to 733.09 , V72.81 and V17.81) and psoriasis (ICD9-CM codes 696.0 to 696.1 ). The Charlson comorbidities index (CCI) was used to score the severity of the comorbidities of patients included in this study.

Outcome measurements. Information regarding patients with cancer was retrieved from ambulance and admission databases. All subjects who received a cancer diagnosis (ICD9-CM codes 140-208) were confirmed by following up from the first diagnosis to their regular treatment. In most cases, patients who receive a cancer diagnosis typically undergo regularly scheduled treatment such as surgery, radiotherapy, chemotherapy and/or drug therapy $(2,14-16)$.

Statistical analysis. The distributions of demographic characteristics, comorbidities and CCI, the incidence of cancer, the duration between the diagnosis of AS and cancer and the survival probability of the patients with AS and the patients without AS were compared; subgroup analyses by gender were also conducted. Differences were examined using the $\chi^{2}$ test for categorical variables and the $t$-test for continuous variables; differences for which $\mathrm{P}<0.05$ were considered to be statistically significant. The standardized incidence ratio (SIR) with a $95 \%$ confidence interval (CI) was used to represent the association between AS and cancer. Cancer-free survival probabilities were computed using the Kaplan-Meier method, and the difference in survival between the AS and non-AS cohorts was tested using the log-rank test; a 2-sided probability value of $\mathrm{P}<0.05$ was considered to be statistically significant. All data were analyzed using SAS 
statistical software version 8.2 (SAS Institute Inc., Cary, NC USA).

\section{Results}

Demographic characteristics. From 6,475 subjects with AS, 1,023 patients who did not satisfy the criteria were excluded; finally, 5,452 patients were enrolled in this study as cases. Therefore, 21,808 subjects without AS were randomly selected as controls and were included in further analyses. The baseline characteristics of patients are presented in Table I. The mean (standard deviation; SD) of the Charlson comorbidity index (CCI) was 1.24 (1.48) for the case group and $0.22(0.59)$ for the control group. The prevalence of other diseases including osteoporosis and psoriasis was significantly higher in the case group compared with the control group $(9.45 \%$ vs. $1.38 \%$; $1.49 \%$ vs. $0.21 \%$, respectively).

Age, sex, comorbidities and cancer risks. The risks of different types of cancer in patients with AS are listed in Table II. The overall risk of cancer was significantly higher in the patients with AS than in the patients without AS (SIR $1.15,95 \%$ CI 1.03-1.27). The AS patients also exhibited a greater risk of developing bone cancer (SIR 3.33, 95\% CI 1.08-7.77), colon cancer (SIR 1.39, 95\% CI 1.03-1.82), prostate cancer (SIR 1.64, 95\% CI 1.04-2.47) or a hematological malignancy (SIR 2.10, 95\% CI 1.32-3.19) than the patients without AS. The male patients with AS were more likely to develop bone cancer (SIR 6.00, 95\% CI 1.23-17.5) and/or prostate cancer (SIR 1.64, 95\% CI 1.04-2.47) than those without AS, whereas female patients with AS had a higher risk of developing colon cancer than non-AS patients (SIR 1.73, 95\% CI 1.19-2.45).

Table III reveals the SIRs for the age of the patient at AS diagnosis, the age of the patient at tumor diagnosis, the follow-up period and the CCI of patients of each sex who were diagnosed with tumors $(n=346)$. The majority of the patients with AS $(n=200,57.8 \%)$ developed cancer within the 3 -year follow-up period. During the first 3 years of follow-up, the risk of developing cancer was higher in patients with AS than in patients without AS in both males (SIR 1.43, 95\% CI 1.17-1.74) and females (SIR 1.55, 95\% CI 1.25-1.89). The risk of cancer was significantly higher in the patients with AS and with a CCI $\geq 2$ compared with the patients without AS (CCI 2-3: SIR 3.70, 95\% CI 3.01-4.75; CCI $\geq 3$ : SIR $11.71,95 \%$ CI 8.40-15.89).

The effect of AS on cancer-free survival. The 2000-2008 cancer-free probabilities of males and females in the two cohorts are presented in Fig. 2A and B, respectively. The results of the log-rank test revealed that the cancer-free probability was significantly lower in the patients with AS than in the patients without AS in both men (3-year log-rank: $\mathrm{P}=0.0207$; 8-year log-rank: $\mathrm{P}=0.0007$; Fig. $2 \mathrm{~A}$ ) and women (3-year log-rank: $\mathrm{P}=0.0004$; 8-year log-rank: $\mathrm{P}<0.0001$; Fig. 2B).

Among the AS patients who developed cancer, compared with matched control patients with cancer, both male (3-year log-rank: $\mathrm{P}=0.0016 ; 8$-year log-rank: $\mathrm{P}=0.0059)$ and female (3-year log-rank: $\mathrm{P}=0.0022 ; 8$-year log-rank: $\mathrm{P}<0.0001$ ) patients with cancer with pre-existing AS exhibited an earlier onset of cancer development compared with cancer patients without AS.

\section{Discussion}

The present study aimed to compare the incidence of cancer in the male and female AS cohorts to determine whether a sex difference exists in the development of certain types of cancer. Based on clinical practice and epidemiology, AS is considered to be a male-predominant disease (2). Thus, identifying gender differences in cancer development may affect treatment policies for clinical practice.

In this large-scale cohort study, it was demonstrated that the male patients with AS experienced an increased risk of bone and prostate cancer and hematological malignancies, whereas the female patients with AS had an increased risk of colon cancer and hematological malignancies. Baecklund et al (17) reported that chronic inflammatory activity within the joints of patients with rheumatoid arthritis increased the risk of lymphoma. The elevation of serum proinflammatory cytokines such as IL-1, TNF- $\alpha$, and IFN- $\gamma$ in patients with AS has been postulated to induce the proliferation of $\mathrm{CD}^{+} \mathrm{T}$ cells $(11,18)$. The presence of inflammation in patients with AS may explain their risk of developing hematological malignancies. For those AS patients treated with TNF inhibitors (TNFi), Mariette et al (19) revealed that TNFi therapy does not affect the overall risk of cancer development in patients with inflammatory arthritis (including those with AS) in a study involving a systematic review and large-scale meta-analysis. However, TNFi therapy appears to increase the risk of skin cancer (19). Raaschou et al (20) demonstrated in a nationwide cohort study that TNFi therapy has no association with the risk of breast cancer recurrence in patients with inflammatory arthritis. In the current study, only 5 of 5452 patients with AS developed skin cancer, and the TNFi factor was not able to be interpreted based on those patients. Furthermore, a genetic linkage to HLA-B27 has been demonstrated to increase the risk of developing hematological malignancies in patients with AS $(21,22)$. An exposure to therapeutic X-rays is considered to increase the risk of hematological cancer in a dose-dependent manner. In the 1950s, radium-224 radiation therapy was used to treat AS; the use of radiation therapy was later found to be associated with the likelihood of developing myeloid leukemia $(22,23)$. Thus, the hematological malignancies observed in this study may be a sequela of radiation therapy.

The present study observed that male patients with AS had a significantly increased risk of bone cancer. AS is a chronic inflammatory disease that affects the interface between cartilage and the mineral plate space. Inflammation surrounding the bone may promote the formation of bone tumors. Osteosarcoma and AS predominantly affect young men (24). However, due to the limited number of patients with bone cancer, additional investigations with a larger number of cases are required to arrive at a more robust conclusion regarding the impact of AS on bone cancer-free survival.

Regarding prostate cancer, clinical observations have revealed that serum androgen levels are highly correlated with the incidence of prostate cancer. In certain studies, serum androgen levels were higher in patients with AS compared with 


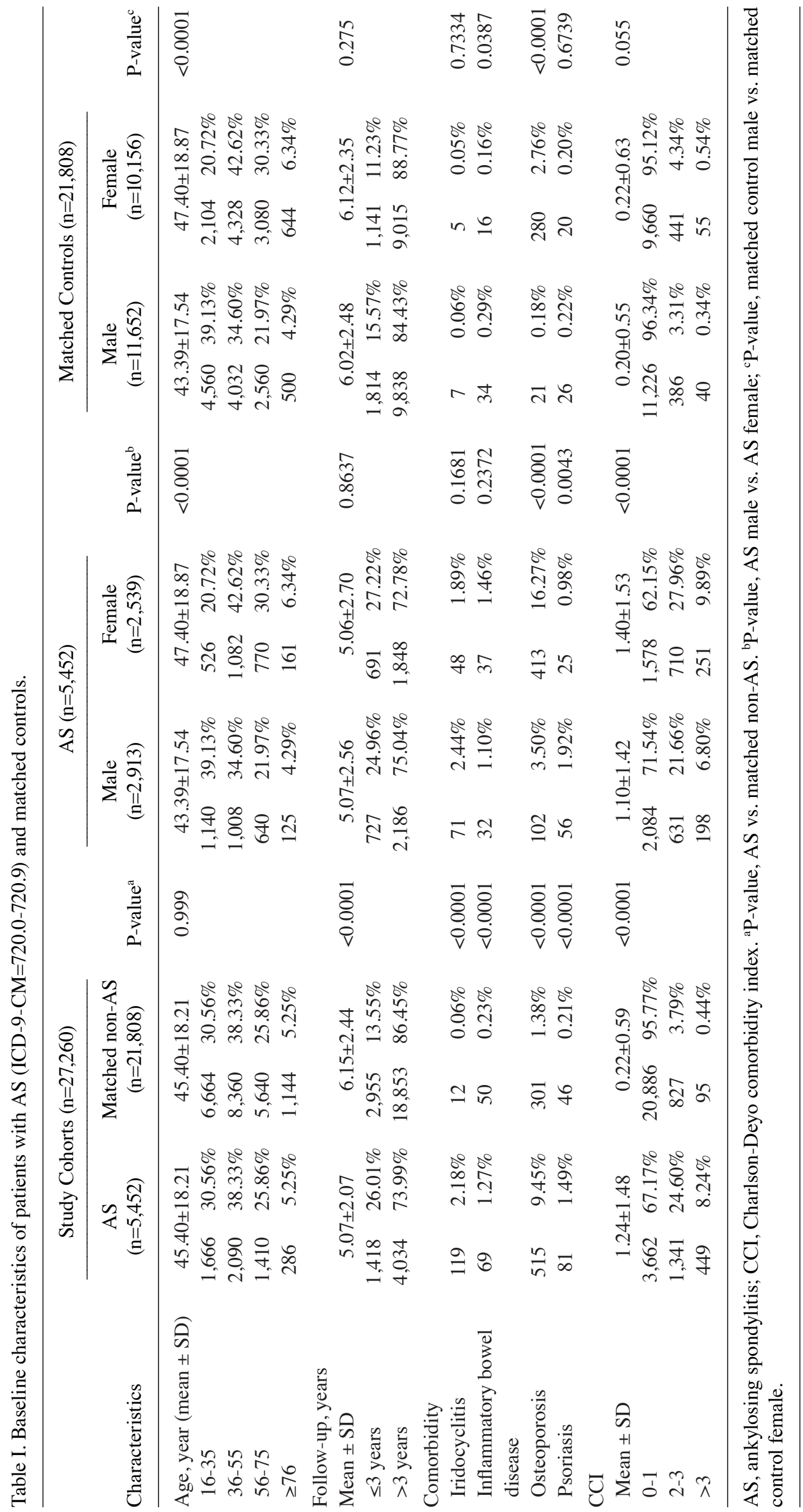




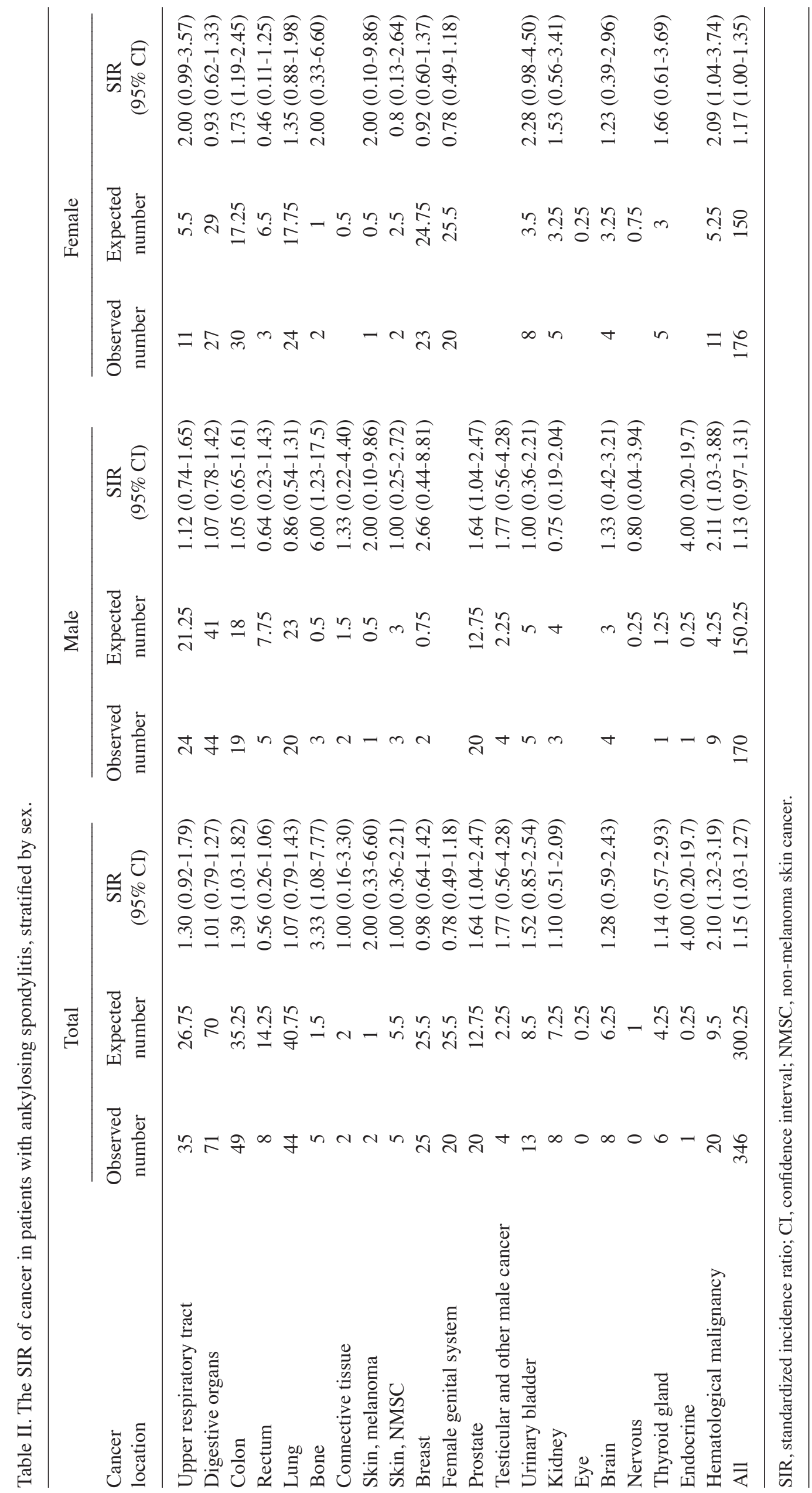




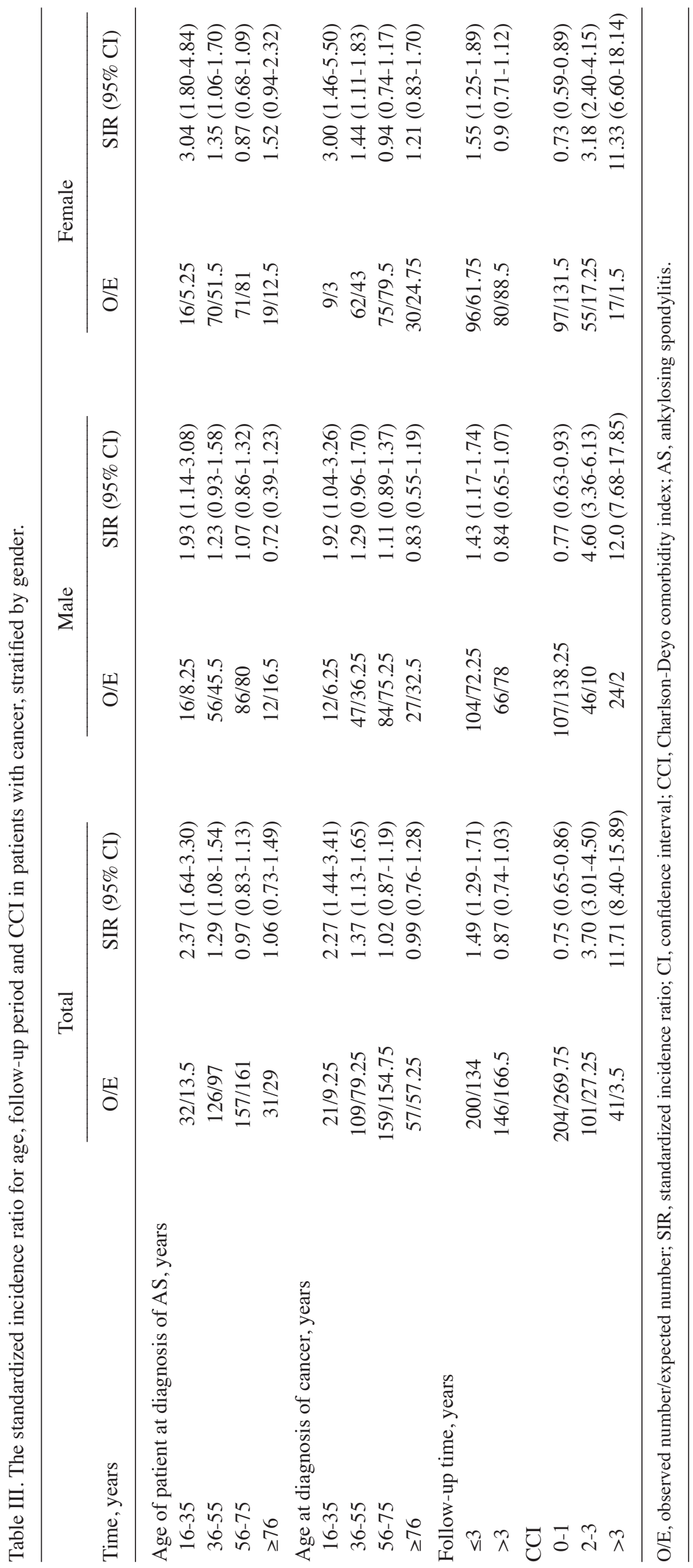


A

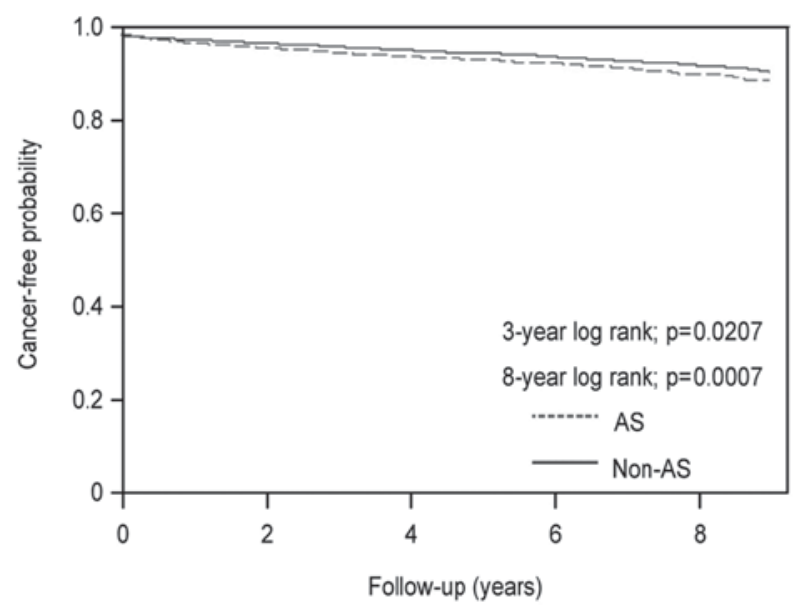

B

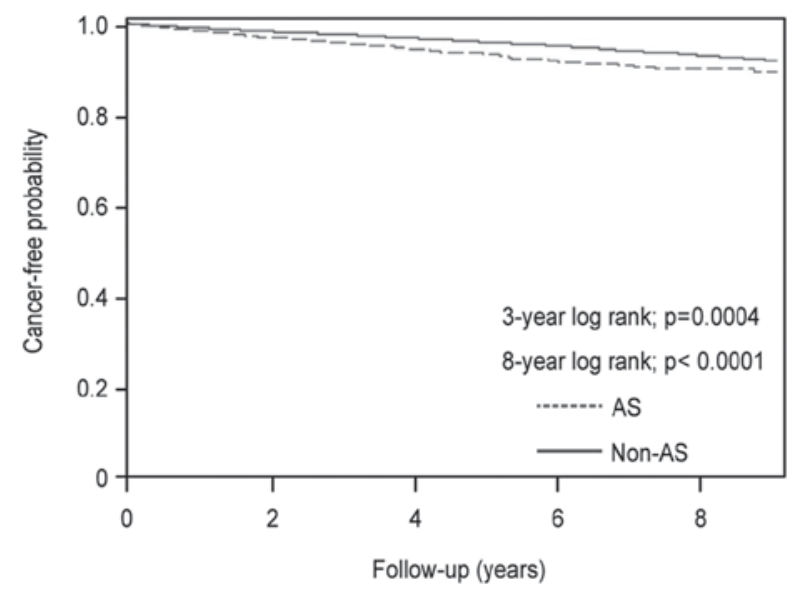

Figure 2. Cancer-free probability in males and females. (A) Ankylosing spondylitis significantly reduced the overall 3- and 8-year cancer-free probabilities in males. (B) Ankylosing spondylitis significantly reduced the overall 3- and 8-year cancer-free probabilities in females.

the healthy population $(25,26)$. Thus, the effect of androgen levels on the incidence of cancer and the potential underlying mechanism by which androgen production affects patients with AS must be further investigated.

Three decades ago, Alcalay et al (27) reported that the HLA-B27 antigen, which has a strong genetic correlation with AS, may act as a carcinogen in colorectal cancer. Additionally, patients with active AS frequently exhibit decreased bone mineral density (28). In this study, the percentage of female patients with AS who also had osteoporosis was significantly higher than the percentage of male patients with AS or patients without AS who had osteoporosis; osteoporosis may therefore increase the risk of colon cancer in female patients with AS. The pathological persistent activation of the immune system by AS triggers immunoglobulin (Ig)A and IgM secretion from the jejunum (29). Inflammatory bowel disease, a disease that shares a complex mixture of genetic and immunological factors with AS, often occurs in patients with AS (30). Circulating colitogenic memory $\mathrm{CD}^{+} \mathrm{T}$ cells alter the microenvironment of the gastrointestinal tract; the immune response in the gut perceives these cells as belonging to benign T-cell leukemia-like tumors (30). Conversely, the use of non-steroidal anti-inflammatory drugs is associated with a reduced risk of colorectal cancer and inflammatory bowel disease $(18,31)$, supporting the hypothesis that activated $\mathrm{CD}^{+} \mathrm{T}$ cells in patients with AS contribute to the formation and progression of gastrointestinal malignancies.

Furthermore, in the present study, the overall 3- and 8-year cancer-free probabilities in patients with AS of both sexes were significantly lower than in those without AS. Furthermore, in patients with AS who developed cancer during the follow-up period, the 3- and 8-year cancer-free probabilities were markedly reduced compared with those in cancer-developing populations who did not have AS. The current data suggest that AS promotes the development of cancer in young adults. Both male and female patients with AS who were younger than 55 years had a higher risk of developing cancer than those who were older. Notably, most malignancies were diagnosed during the first 3 years following the diagnosis of AS, and a CCI of $\geq 2$ significantly increased the risk of cancer in patients with AS (Table III). These observations suggest that both AS and severe comorbidities accelerate tumorigenesis.

In another study, Feltelius et al (15) concluded that AS does not affect the overall cancer risk in Sweden. In previous studies reported by Hemminki et al AS patients in Sweden showed an increased risk for multiple myeloma (32), a decreased risk for digestive tract cancer (33), and no altered risk for non-Hodgkin lymphoma (34). In female cancer types of the breast, uterus, ovary and other genital organs, a low SIR in female patients with AS was noted (35). In the current study, AS increased the overall risk for hematological malignancies. In addition, a significant difference was not identified in the risk of female cancer (breast cancer and cancer involving the female genital system) in patients with AS, indicating that a racial difference between Caucasian and Chinese patients is likely a primary factor regulating cancer risk among patients with AS. Using a Cox proportional hazards regression model, Sun et al (36) reported that patients with AS had an increased cancer incidence, but a potential sex difference was not confirmed. The results from the cancer type subgroupings in the present study differed from those reported by Sun et al (36). Additionally, a longer recruitment period and a larger number of patients compared with Sun et al were used in the current study, indicating that the results from the present study are more representative of the true prevalence in Taiwan.

The strengths of the present study include the large sample size and the lengthy follow-up period. However, there were specific limitations. In this cohort study, bias may have resulted from the fact that $1 \%$ of the Taiwanese population is not included in the NHIRD. Information on disease severity, biochemical test results, the levels of circulating chemokines, the level of medical attention, the socioeconomic status of subjects and the HLA characteristics of the patients with AS are not reported in the database. For an extremely rare cancer, an increase in the number of new cases of that cancer will affect the outcome of the data analysis. However, despite these 
limitations, our study provides evidence of cancer risks and sex differences among patients with AS. A large-scale, elaborate, prospective cohort study is required to confirm these findings and strengthen these hypotheses.

In conclusion, AS in combination with severe comorbidities is a risk factor for the development of cancer in males and females. Tumor screening during the first 3 years following the diagnosis of AS provides a chance for early detection of hematological malignancies in males and females, prostate cancer in males, and colon cancer in females; young patients with AS and patients with a CCI value $\geq 2$ are particularly at risk. Due to the tendency to develop cancer and sex differences, cancer screening in patients with AS is encouraged in clinical practice.

\section{Acknowledgements}

The authors acknowledge the support of research grants from the National Science Council (grant nos.NSC101-2314-B-038-022-MY3,NSC101-2120-M-010-002, NSC101-2911-I-009-101 and NSC100-2911-I-010-503). The authors also acknowledge the financial support from Wan Fang Hospital, Taipei Medical University (grant nos. 104swf03 and MOST 104-2314-B-038 -013-MY3), and Shuang Ho Hospital, Taipei Medical University (grant no. 103TMU-SHH-10). The authors wish to thank Dr. Yen-Kuang Lin (Research Center of Biostatistics, College of Management, Taipei Medical University) for assistance in conducting this research.

\section{References}

1. Braun J and Sieper J: Ankylosing spondylitis. Lancet 369: 1379-1390, 2007.

2. Chen HH, Chen TJ, Chen YM, Ying-Ming C and Chen DY: Gender differences in ankylosing spondylitis-associated cumulative healthcare utilization: A population-based cohort study. Clinics (Sao Paulo) 66: 251-254, 2011

3. Ho HH and Chen JY: Ankylosing spondylitis: Chinese perspective, clinical phenotypes, and associated extra-articular systemic features. Curr Rheumatol Rep 15: 344, 2013.

4. Selmi C: Diagnosis and classification of autoimmune uveitis Autoimmun Rev 13: 591-594, 2014.

5. Bayen H, Bayen MC, De Curzon HP, Espinasse-Berrod MA, Manderieux N, Furia M and Campinchi R: Involvement of the posterior eye segment in HLA B27(+) iridocyclitis. Incidence. Value of surgical treatment. J Fr Ophtalmol 11: 561-566, 1988 (In French).

6. Davey-Ranasinghe N and Deodhar A: Osteoporosis and vertebral fractures in ankylosing spondylitis. Curr Opin Rheumatol 25: 509-516, 2013.

7. Atzeni F, Defendenti C, Ditto MC, Batticciotto A, Ventura D, Antivalle M, Ardizzone S and Sarzi-Puttini P: Rheumatic manifestations in inflammatory bowel disease. Autoimmun Rev 13: 20-23, 2014.

8. Siegel R, Naishadham D and Jemal A:Cancer statistics for Hispanics/Latinos, 2012. CA Cancer J Clin 62: 283-298, 2012.

9. Mantovani A, Allavena P, Sica A and Balkwill F: Cancer-related inflammation. Nature 454: 436-444, 2008.

10. Balkwill $\mathrm{F}$ and Mantovani A: Inflammation and cancer: Back to Virchow? Lancet 357: 539-545, 2001.

11. Hreggvidsdottir HS, Noordenbos T and Baeten DL: Inflammatory pathways in spondyloarthritis. Mol Immunol 57: 28-37, 2014.

12. Iqbal U, Nguyen PA, Syed-Abdul S, Yang HC, Huang CW, Jian WS, Hsu MH, Yen Y and Li YC: Is long-term use of benzodiazepine a risk for cancer? Medicine 94: e483, 2015.

13. WHO International Classification of Disease codes. http://www. who.int/classifications/icd/en/. Accessed November 17, 2016.

14. Hwang CY, Chen YJ, Lin MW, Chen TJ, Chu SY, Chen CC, Lee DD, Chang YT, Wang WJ and Liu HN: Cancer risk in patients with allergic rhinitis, asthma and atopic dermatitis: A nationwide cohort study in Taiwan. Int J Cancer 130: 1160-1175, 2012.
15. Feltelius N, Ekbom A and Blomqvist P: Cancer incidence among patients with ankylosing spondylitis in Sweden 1965-95: A population based cohort study. Ann Rheum Dis 62: 1185-1188, 2003.

16. Janssen F and Kunst AE: ICD coding changes and discontinuities in trends in cause-specific mortality in six European countries, 1950-99. Bull World Health Organ 82: 904-913, 2004.

17. Baecklund E, Ekbom A, Sparén P, Feltelius N and Klareskog L: Disease activity and risk of lymphoma in patients with rheumatoid arthritis: Nested case-control study. BMJ 317: 180-181, 1998.

18. Kanai T, Nemoto Y, Tomita T, Totsuka T, Watanabe $M$ and Hibi T: Persistent retention of colitogenic $\mathrm{CD}^{+}$memory $\mathrm{T}$ cells causes inflammatory bowel diseases to become intractable. Inflamm Bowel Dis 15: 926-934, 2009.

19. Mariette X, Matucci-Cerinic M, Pavelka K, Taylor P, van Vollenhoven R, Heatley R, Heatley R, Walsh C, Lawson R, Reynolds A and Emery P: Malignancies associated with tumour necrosis factor inhibitors in registries and prospective observational studies: A systematic review and meta-analysis. Ann Rheum Dis 70: 1895-1904, 2011.

20. Raaschou P, Frisell T and Askling J; ARTIS Study Group: TNF inhibitor therapy and risk of breast cancer recurrence in patients with rheumatoid arthritis: A nationwide cohort study. Ann Rheum Dis 74: 2137-2143, 2015.

21. Au WY, Hawkins BR, Cheng N, Lie AK, Liang R and Kwong YL: Risk of haematological malignancies in HLA-B27 carriers. Br J Haematol 115: 320-322, 2001.

22. Wick RR, Nekolla EA, Gaubitz M and Schulte TL: Increased risk of myeloid leukaemia in patients with ankylosing spondylitis following treatment with radium-224. Rheumatology (Oxford) 47: 855-859, 2008

23. Weiss HA, Darby SC, Fearn T and Doll R: Leukemia mortality after X-ray treatment for ankylosing spondylitis. Radiat Res 142: $1-11,1995$.

24. Raymond AK and Jaffe N: Osteosarcoma multidisciplinary approach to the management from the pathologist's perspective. Cancer Treat Res 152: 63-84, 2009.

25. Spector TD, Ollier W, Perry LA, Silman AJ, Thompson PW and Edwards A: Free and serum testosterone levels in 276 males: A comparative study of rheumatoid arthritis, ankylosing spondylitis and healthy controls. Clin Rheumatol 8: 37-41, 1989.

26. Gordon D, Beastall GH, Thomson JA and Sturrock RD: Androgenic status and sexual function in males with rheumatoid arthritis and ankylosing spondylitis. Q J Med 60: 671-679, 1986.

27. Alcalay M, Bontoux D, Maire P, Matuchansky C, Alcalay D and Tanzer J: HLA-B27 and colorectal cancer. N Engl J Med 307: 443-444, 1982.

28. Singh HJ, Nimarpreet K, Ashima, Das S, Kumar A and Prakash S: Study of bone mineral density in patients with ankylosing spondylitis. J Clin Diagn Res 7: 2832-2835, 2013.

29. Feltelius N, Hvatum M, Brandtzaeg P, Knutson L and Hällgren R: Increased jejunal secretory $\operatorname{IgA}$ and $\operatorname{IgM}$ in ankylosing spondylitis: Normalization after treatment with sulfasalazine. J Rheumatol 21: 2076-2081, 1994.

30. Van Praet L, Jacques P, Van den Bosch F and Elewaut D: The transition of acute to chronic bowel inflammation in spondyloarthritis. Nat Rev Rheumatol 8: 288-295, 2012.

31. Kanai T, Watanabe $M$ and Hibi T: Systemically circulating colitogenic memory $\mathrm{CD}^{+} \mathrm{T}$ cells may be an ideal target for the treatment of inflammatory bowel diseases. Keio J Med 58: 203-209, 2009.

32. Hemminki K, Liu X, Försti A, Ji J, Sundquist J and Sundquist K: Effect of autoimmune diseases on incidence and survival in subsequent multiple myeloma. J Hematol Oncol 5: 59, 2012.

33. Hemminki K, Liu X, Ji J, Sundquist J and Sundquist K: Autoimmune disease and subsequent digestive tract cancer by histology. Ann Oncol 23: 927-933, 2012.

34. Fallah M, Liu X, Ji J, Försti A, Sundquist K and Hemminki K: Autoimmune diseases associated with non-Hodgkin lymphoma: A nationwide cohort study. Ann Oncol 25: 2025-2030, 2014.

35. Hemminki K, Liu X, Ji J, Försti A, Sundquist J and Sundquist K: Effect of autoimmune diseases on risk and survival in female cancers. Gynecol Oncol 127: 180-185, 2012.

36. Sun LM, Muo CH, Liang JA, Chang SN, Sung FC and Kao CH: Increased risk of cancer for patients with ankylosing spondylitis: A nationwide population-based retrospective cohort study. Scand J Rheumatol 43: 301-306, 2014. 